\title{
Fatty acid amide hydrolase binding is inversely correlated with amygdalar functional connectivity: a combined positron emission tomography and magnetic resonance imaging study in healthy individuals
}

\author{
Duncan G.J. Green, HBSc*; Jinhee Kim, PhD*; Stephen J. Kish, PhD; \\ Rachel F. Tyndale, PhD; Matthew N. Hill, PhD; Antonio P. Strafella, MD, PhD; \\ Junchao Tong, PhD; Tina McCluskey, MSc; Duncan J. Westwood, HBSc; \\ Sylvain Houle, MD, PhD; Nancy J. Lobaugh, PhD; Isabelle Boileau, PhD
}

\begin{abstract}
Background: Upregulation of the endocannabinoid enzyme fatty acid amide hydrolase (FAAH) has been linked to abnormal activity in frontoamygdalar circuits, a hallmark of posttraumatic stress disorder. We tested the hypothesis that FAAH levels in the amygdala were negatively correlated with functional connectivity between the amygdala and prefrontal cortex, subserving stress and affect control. Methods: Thirty-one healthy participants completed positron emission tomography (PET) imaging with the FAAH probe [C-11]CURB, and resting-state functional MRI scans. Participants were genotyped for the FAAH polymorphism rs324420, and trait neuroticism was assessed. We calculated amygdala functional connectivity using predetermined regions of interest (including the subgenual ventromedial prefrontal cortex [sgvmPFC] and the dorsal anterior cingulate cortex [dACC]) and a seed-to-voxel approach. We conducted correlation analyses on functional connectivity, with amygdala [C-11]CURB binding as a variable of interest. Results: The strength of amygdala functional connectivity with the sgvmPFC and dACC was negatively correlated with [C-11]CURB binding in the amygdala (sgvmPFC: $r=-0.38, q=0.04$; dACC: $r=-0.44 ; q=0.03$ ). Findings were partly replicated using the seed-to-voxel approach, which showed a cluster in the ventromedial prefrontal cortex, including voxels in the dACC but not the sgvmPFC (cluster-level, family-wise error rate corrected $p<0.05$ ). Limitations: We did not replicate earlier findings of a relationship between an FAAH polymorphism (rs324420) and amygdala functional connectivity. Conclusion: Our data provide preliminary evidence that lower levels of FAAH in the amygdala relate to increased frontoamygdalar functional coupling. Our findings were consistent with the role of FAAH in regulating brain circuits that underlie fear and emotion processing in humans.
\end{abstract}

\section{Introduction}

Emotion dysregulation - characterized by the inability to manage negative emotions such as fear, anger or sadness is a common symptom of many mental health disorders. Elevated amygdala hyperactivity has been linked with emotion dysregulation and trait neuroticism (a tendency to experience anxiety, fear and depressed mood), ${ }^{1}$ and is a risk phenotype for psychiatric disorders, including posttraumatic stress disorder (PTSD). ${ }^{2,3}$ Mounting evidence suggests that the endo- cannabinoid system - and in particular one of its key enzymes, fatty acid amide hydrolase (FAAH) - is critically involved in emotion regulation and fear processing. ${ }^{4}$ In this study we investigated the relationship between brain levels of the enzyme FAAH and functional connectivity in the amygdalar networks that regulate stress and affect.

Fatty acid amide hydrolase is the primary catabolic enzyme for the major endocannabinoid $\mathrm{N}$-arachidonoylethanolamine (anandamide, AEA) and for other ethanolamides, including oleoylethanolamine and palmitoylethanolamide.

Correspondence to: I. Boileau, Centre for Addiction and Mental Health, 250 College St., Toronto, ON, M5T 1R8; isabelle.boileau@camh.ca

*These authors contributed equally to this work.

Submitted Jan. 22, 2020; Revised Jul. 3, 2020; Accepted Sept. 29, 2020

DOI: 10.1503/jpn.200010 
A cannabimimetic eicosanoid, AEA signals via the cannabinoid type 1 (CB1) receptor; efforts to increase its levels in brain by blocking FAAH may have therapeutic potential. ${ }^{5}$ In this regard, recent work has demonstrated that pharmacological inhibition of FAAH in humans can reduce physiologic and behavioural stress responses and enhance fear extinction. ${ }^{6}$

Reduced FAAH activity can be inherited through a single nucleotide polymorphism (rs324420, C385A, P129T) which destabilizes the FAAH enzyme. ${ }^{7}$ The minor A allele (A/A homozygotes and $\mathrm{A} / \mathrm{C}$ heterozygotes) is found in $38 \%$ of people of European descent, ${ }^{8}$ who consequently have lower FAAH binding in brain. ${ }^{9}$ Genetic association studies have found that people with the C385A minor allele are less sensitive to pain and cold and have lower trait anxiety. ${ }^{2,10}$ This association has also recently been made in the remarkable case report of a woman who inherited both the FAAH C385A hypomorphic allele and a microdeletion in a $F A A H$ pseudogene and lives without pain or worry. ${ }^{11}$ Preclinical studies using pharmacological manipulations and gene knockout mice have also suggested that an elevation in AEA through blockade of FAAH decreases nociception, anxiety, fear responses and amygdala neuron excitability to threat. ${ }^{5}$

Echoing preclinical findings, ${ }^{5}$ some human fMRI studies have shown that people with the FAAH C385A minor allele variant have blunted amygdala reactivity ${ }^{12}$ and increased temporal habituation of amygdala activity ${ }^{13}$ to threat; they express low trait anxiety and stress reactivity compared with $\mathrm{C} / \mathrm{C}$ homozygotes. ${ }^{2}$ Both functional and structural connectivity studies have shown an association between the FAAH rs324420 single nucleotide polymorphism and both frontolimbic structural connectivity ${ }^{14}$ and amygdala restingstate functional connectivity. ${ }^{15,16}$ Specifically, compared with $\mathrm{C} / \mathrm{C}$ homozygotes, people with the A allele have greater frontolimbic structural connectivity (based on diffusion tensor imaging $)^{14}$ and functional connectivity, ${ }^{15,16}$ but not taskrelated effective connectivity, ${ }^{15}$ between the amygdala and medial prefrontal regions. Functional connectivity studies have so far been inconsistent with respect to the regional extent of their findings: one study showed greater coupling with the subgenual ventromedial prefrontal cortex (sgvmPFC) $)^{16}$ and the other with the dorsal anterior cingulate cortex (dACC).$^{15}$ Although both of these regions are chiefly involved in emotion and fear processing, they are believed to have distinct roles. For example, the sgvmPFC and $\mathrm{dACC}$ have been involved in the generation and appraisal of emotional responses, respectively. But they have been involved differently in the acquisition and expression of conditioned fear; the sgvmPFC has a presumed role in conditioned aversion, whereas the dACC is involved in the cognitive-affective aspects of fear and pain. ${ }^{17,18}$ In the current study, we investigated functional connectivity in both of these frontolimbic circuits.

We developed [C-11-carbonyl]-6-hydroxy-[1,10biphenyl]-3-yl cyclohexylcarbamate ([C-11]CURB), the first available positron emission tomography (PET) radiotracer for imaging FAAH. ${ }^{19}$ Kinetic analysis of [C-11]CURB indicates that the composite parameter $\lambda \mathrm{k}_{3}\left(\lambda=\mathrm{K}_{1} / \mathrm{k}_{2}\right)$, de- rived from a 2-tissue compartment model for irreversible trapping, is a robust index of FAAH levels ${ }^{20}$ with strong test-retest reliability $(<10 \%){ }^{21}$

We combined [C-11]CURB PET imaging with resting-state functional MRI in healthy participants to examine whether amygdala [C-11]CURB binding was related to amygdala functional connectivity. Based on previous literature showing greater functional connectivity between the amygdala and the sgvmPFC or dACC in people with the FAAH C385A single nucleotide polymorphism, ${ }^{15,16}$ we hypothesized that lower [C-11]CURB binding in the amygdala would be correlated with stronger functional connectivity between the amygdala and the sgvmPFC and dACC. We explored whether trait neuroticism was related to functional connectivity and [C-11]CURB binding.

\section{Methods}

\section{Participants}

We recruited 34 participants using online advertisements. They provided written informed consent to complete a [C-11] CURB PET and an MRI scan as part of 2 studies approved by the Centre for Addiction and Mental Health Research Ethics Board. The [C-11]CURB PET data from 20 participants have been published previously. ${ }^{9,22}$ Participants were excluded if they had significant medical conditions, neurologic illnesses, head trauma, Axis I psychiatric disorders as per the Structured Clinical Interview for DSM-IV, ${ }^{23}$ or MRI and PET contraindications (including pregnancy). We used urine testing on scan days (Rapid Response BTNX Inc.) and scalp hair samples at screening (United States Drug Testing Laboratories) to exclude participants with recent drug use. All participants were asked to complete the NEO Personality Inventory-Revised to assess trait neuroticism. We genotyped FAAH C385A rs324420 according to published procedures, because it affects [C-11] CURB quantification. ${ }^{9}$

\section{PET image acquisition and reconstruction}

We took urine samples to rule out pregnancy and recent drug use. We also assessed recent alcohol and tobacco use, determined by breath-alcohol and expired carbon monoxide (> 10 ppm) measurements, respectively, before the PET scan. Smokers were asked not to smoke on the day of the scan.

[C-11]CURB radiosynthesis has been described previously. ${ }^{24}$ We performed PET scans on an HRRT brain tomograph (CPS/Siemens) as described in Rusjan and colleagues. ${ }^{20}$ Briefly, participants received an injection of $342 \pm$ $33 \mathrm{MBq}$ (mean \pm standard deviation) of [C-11]CURB while supine, with their head secured by a thermoplastic mask. We then acquired emission data for 1 hour in sequential frames of increasing duration. ${ }^{9}$ Images were reconstructed from $2 \mathrm{D}$ sinograms with a $2 \mathrm{D}$ filtered-back projection algorithm, using a HANN filter at Nyquist cut-off frequency. We counted radioactivity in arterial blood with an automatic blood sampling system (Model PBS-101, Veenstra Instruments) for the first 22.5 minutes after injection. We measured 
radioactivity in plasma and metabolization from arterial blood samples extracted at 3, 7, 12, 20, 30, 45 and 60 minutes after injection. We generated a metabolite-corrected plasma curve and used it as the input function for the kinetic analysis. ${ }^{20} \mathrm{We}$ interpolated blood-to-plasma radioactivity ratios using a biexponential function, and the parent plasma fraction using a Hill function.

\section{PET image analyses}

We extracted time-activity curves in the amygdala and 10 other ROIs (hippocampus, occipital cortex, parietal cortex, medial prefrontal cortex, prefrontal cortex, cingulate cortex, temporal cortex, ventral striatum, dorsal striatum and thalamus) using ROMI, as described in Rusjan and colleagues. ${ }^{25}$ We analyzed regional time-activity curves using a 2-tissue compartment model for irreversible trapping as described in Rusjan and colleagues, ${ }^{20}$ generating regional $\lambda \mathrm{k}_{3}\left(\lambda \mathrm{k}_{3}=\mathrm{k}_{3}\right.$ $\times \mathrm{K}_{1} / \mathrm{k}_{2}$ ), the outcome measure for FAAH levels. Amygdala $\lambda \mathrm{k}_{3}$ was the variable of interest in the current study.

\section{MRI data acquisition}

We acquired MRI data using a 3T GE Discovery MR750 with an 8-channel radiofrequency head coil (General Electric). We obtained proton-density images for PET coregistration and region of interest (ROI) delineation (spin echo imaging; repetition time $=$ minimum $/$ full,$\sim 6 \mathrm{~s}$; echo time $=13.7 \mathrm{~ms}$; echo train length $=8$; receiver bandwidth $\pm 15.6 \mathrm{kHz}$; field of view $=22 \mathrm{~cm}$; matrix size $=256 \times 256$; slice thickness $=$ $2 \mathrm{~mm}$ ). We obtained sagittal $T_{1}$-weighted structural MRIs for resting-state functional MRI coregistration and ROI delineation (repetition time $=6.7 \mathrm{~ms}$; echo time $=3 \mathrm{~ms}$; inversion time $=600 \mathrm{~ms}$; flip angle $=8^{\circ}$; field of view $=230 \times 230 \mathrm{~mm}$; matrix size $=256 \times 256 \times 200$; voxel size $\left.=0.9 \mathrm{~mm}^{3}\right)$. We obtained resting-state functional MRI data over 7 minutes using a $T_{2}{ }^{*}$-weighted spiral-in and -out $2 \mathrm{D}$ gradient echo sequence (repetition time $=2500 \mathrm{~ms}$; echo time $=30 \mathrm{~ms}$; flip angle $=70^{\circ} ; 39$ sequential slices with no gap; field of view = $200 \times 200 \mathrm{~mm}$; matrix size $=64 \times 64$; slice thickness $=$ $3.0 \mathrm{~mm}$; voxel size $=3.1 \times 3.1 \times 3.0 \mathrm{~mm}) .{ }^{26}$ We obtained a total of 168 volumes per scan.

\section{Resting-state fMRI analyses}

We conducted analyses using FMRIB Software Library (FSL; www.fmrib.ox.ac.uk/fsl), Statistical Parametric Mapping 12 (SPM12; www.fil.ion.ucl.ac.uk/spm) and the Functional Connectivity toolbox (CONN v17f; www.nitrc.org/projects/conn, RRID:SCR_009550). The first 4 volumes of each scan were discarded for magnetization equilibrium, leaving 164 volumes for analysis. We removed MRI sequence-specific artifacts using the ICA-based denoising tool (MELODIC) in FSL, separately on the spiral-in and spiral-out images. We removed artifact-related components using the FIX toolbox in FSL. ${ }^{27}$ This procedure included the automated removal of non-brain tissue $\left(\mathrm{BET}^{28}\right.$ ) and head-motion correction (MCFLIRT $\left.{ }^{29}\right)$. We compared the cleaned spiral-in and spiral-out functional images with a weighted average ${ }^{26}$ We performed further preprocessing steps of the combined functional images in the CONN toolbox. We identified outlier volumes for global signal intensity and head motion using Artifact Detection Tools (ART; with 5 for $z$-score scan-to-scan global signal changes and 0.9 for scan-to-scan head-motion composite changes) as implemented in CONN, and we used them as the confounds in the post-processing steps. We normalized the functional data using affine transformation to the SPM echo planar imaging template in standard space, followed by nonlinear registration to the echo planar imaging template and resampling to $2 \mathrm{~mm}$ isotropic voxels using fourth-order spline interpolation. ${ }^{30}$ We then spatially smoothed the normalized resting-state functional MRI data with a $6 \mathrm{~mm}$ full width at half maximum Gaussian kernel. The post-processing steps included regression of confounds, linear detrending and bandpass filtering $(0.008-0.09 \mathrm{~Hz})$, performed simultaneously as implemented in CONN. Specifically, the 6 head-motion parameters with first-order derivatives were included. We obtained 5 principal components from the mean blood-oxygenlevel-dependent time series of white matter and cerebrospinal fluid time series using the CompCor method. ${ }^{31}$

We calculated the mean frame-wise displacement value using the method described in Van Dijk and colleagues, ${ }^{32}$ and 3 participants with a mean frame-wise displacement greater than $0.3 \mathrm{~mm}$ were excluded from further analysis. We subjected the resulting resting-state functional MRI data of 31 participants to functional connectivity analysis.

\section{Functional connectivity: ROI-to-ROI approach}

Our primary aim was to test the hypothesis that lower amygdalar [C-11]CURB $\lambda \mathrm{k}_{3}$ would correlate with stronger functional connectivity between the amygdala and the sgvmPFC and dACC. The approach in the following section replicates the methodology described in Gärtner and colleagues ${ }^{15}$ and Dincheva and colleagues. ${ }^{16}$

We created the anatomic bilateral amygdala mask using Wake Forest University PickAtlas software. ${ }^{33}$ We created spherical prefrontal ROI masks with a radius of $4 \mathrm{~mm}$ centred on the coordinates derived from Dincheva and colleagues. ${ }^{16}$ Specifically, the prefrontal ROIs included the sgvmPFC (Montreal Neurological Institute [MNI] coordinates $x, y, z=0,40,-3)$ and dACC $(x, y, z=-3,25,19)$. Temporal signal-to-noise ratio calculated with FSL suggested that ROI masks were of sufficient size. We defined functional connectivity strength as the bivariate Pearson correlation coefficient $r$ of the 2 time series. We calculated amygdalasgvmPFC and amygdala-dACC functional connectivity at the participant level and converted it to z-scores using the Fisher $r$-to- $z$ transformation, which we in turn subjected to group-level correlation analysis with amygdalar FAAH $\lambda \mathrm{k}_{3}$.

\section{Functional connectivity: seed-to-voxel approach}

Applying the amygdala mask used in the ROI-to-ROI analysis, we also obtained whole-brain amygdala functional connectivity maps using a seed-to-voxel approach and identified 
regions over the whole brain in which functional connectivity with the amygdala was related to the amygdala [C-11]CURB $\lambda \mathrm{k}_{3 .}$. At the participant level, we extracted the averaged blood-oxygen-level-dependent time series of the amygdala from all voxels in the amygdala seed ROI. We carried out linear regression analysis between the amygdala time series and the time series of the rest of the brain in a voxel-wise manner, resulting in a functional connectivity $\beta$ map in which each voxel's value represented its linear association with the amygdalar time series. The individual functional connectivity maps were then transformed to a $z$-score map using the Fisher $r$-to- $z$ transformation and were entered into a grouplevel whole-brain analysis to examine amygdala functional connectivity associated with amygdala [C-11]CURB $\lambda \mathrm{k}_{3}$.

\section{Statistical analysis}

For the ROI-to-ROI approach, we performed a Pearson product moment correlation analysis to test the relationship between amygdala [C-11]CURB $\lambda \mathrm{k}_{3}$ and the strength of amygdala-sgvmPFC and amygdala-dACC functional connectivity, similar to the analysis used in Gärtner and colleagues ${ }^{15}$ and Dincheva and colleagues. ${ }^{16}$ We performed statistical analysis using SPSS (IBM SPSS Statistics 24), and statistical significance was determined using a false discovery rate (FDR)-adjusted $p$ value (or $q$ value) of 0.05 .

For the seed-to-voxel approach, we performed a wholebrain linear regression analysis with [C-11]CURB $\lambda \mathrm{k}_{3}$ in the amygdala as an independent variable and amygdala functional connectivity maps as the dependent variables. The statistical significance threshold was set at a voxel-level $p<0.001$ and corrected (cluster-level family-wise error rate [cFWE]) to $p_{\mathrm{cFWE}}<0.05$ for multiple comparisons using Gaussian random field theory ${ }^{34}$ as implemented in the CONN toolbox. We used individual mean frame-wise displacement as a group-level motion covariate to further control for the influence of potential head movement on functional connectivity. ${ }^{32}$ All significant results are reported in MNI space.

We conducted covariate analyses using partial correlations. We chose the covariates based on the reported effects of age, ${ }^{14,35}$ sex, ${ }^{36}$ body mass index, ${ }^{37}$ nicotine $^{38}$ and the FAAH C385A single nucleotide polymorphism (which is related to ethnicity) ${ }^{8}$ on FAAH activity. We conducted Pearson product moment correlation analyses to investigate whether trait neuroticism (NEO Personality Inventory-Revised total) was correlated with functional connectivity and amygdala [C-11]CURB $\lambda \mathrm{k}_{3}$.

We used $t$ tests and analyses of covariance to investigate differences in functional connectivity and trait neuroticism between $F A A H$ genotype groups $(\mathrm{C} / \mathrm{C}$ v. A/A and $\mathrm{A} / \mathrm{C})$.

\section{Results}

Demographic information for the 31 participants with usable data are outlined in Table 1 . Three of the 34 participants were excluded: 1 owing to excessive head movement (mean framewise displacement $>0.3 \mathrm{~mm}$ ) and 2 for poor MRI quality due to spiral artifact. Five of the remaining 31 participants did not complete the NEO Personality Inventory-Revised.

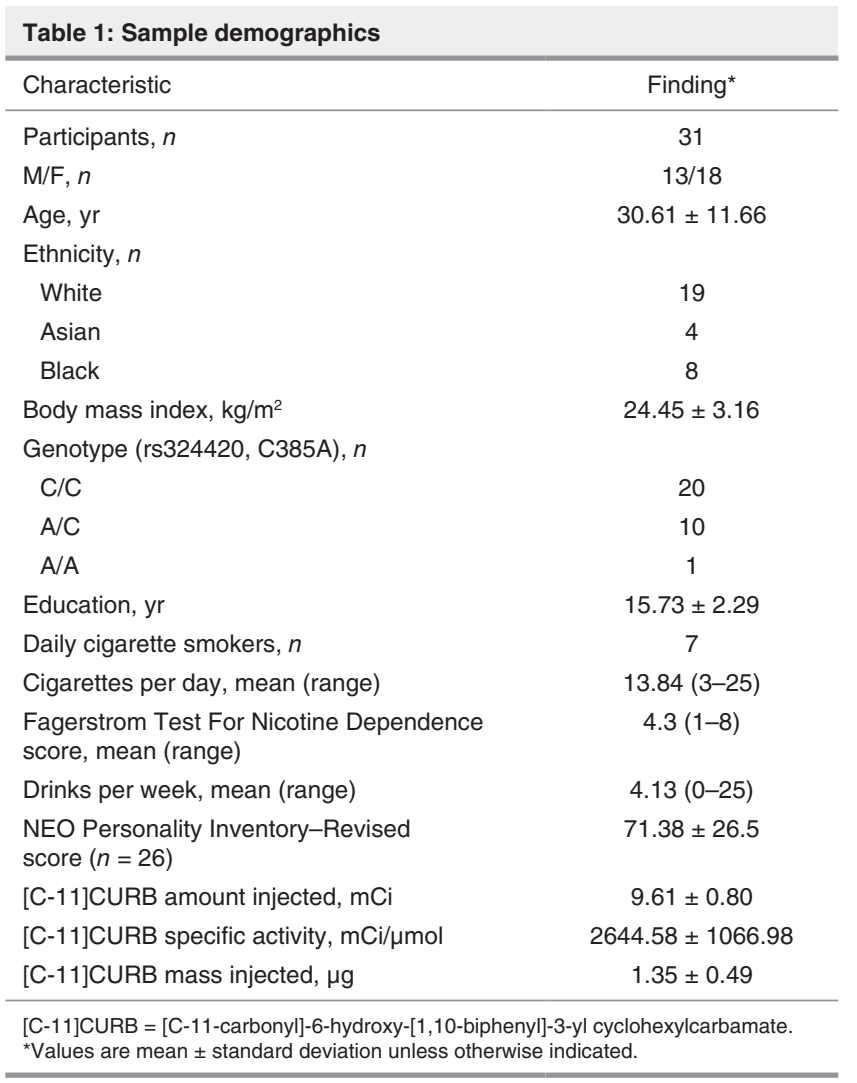

Whole brain functional connectivity maps of the bilateral amygdala seed region are reported in Appendix 1, Figure S1, available at jpn.ca/200010-a1.

\section{ROI-to-ROI approach}

We first tested the relationship between [C-11]CURB $\lambda \mathrm{k}_{3}$ in the amygdala and amygdalar functional connectivity with 2 a priori brain regions: the sgvmPFC and the dACC. Using a ROI-to-ROI approach, we found a significant negative correlation between amygdala [C-11]CURB $\lambda \mathrm{k}_{3}$ and amygdala-sgvmPFC functional connectivity $(r=-0.38 ; q=$ 0.04; Figure 1A). We also found a significant negative correlation between amygdala [C-11]CURB $\lambda \mathrm{k}_{3}$ and amygdala-dACC functional connectivity $(r=-0.44 ; q=$ 0.03; Figure 1B). Trait neuroticism was not related to amygdala functional connectivity with the ROIs (sgvmPFC: $r=$ $-0.12 ; p=0.28$; dACC $r=-0.29 p=0.08$ ) or to the amygdala [C-11]CURB $\lambda \mathrm{k}_{3}(r=0.20 ; p=0.17)$.

\section{Seed-to-voxel approach}

As seen in Figure 2, a seed-to-voxel analysis further confirmed that amygdala [C-11]CURB $\lambda \mathrm{k}_{3}$ was negatively correlated with functional connectivity between the amygdala and a cluster that included the ventromedial prefrontal cortex (vmPFC) and the dACC (Brodmann areas [BAs] 24 and 32; MNI coordinates $x, y, z=14,40,10 ; k=280 ; t=6.67 ; z=$ 
A

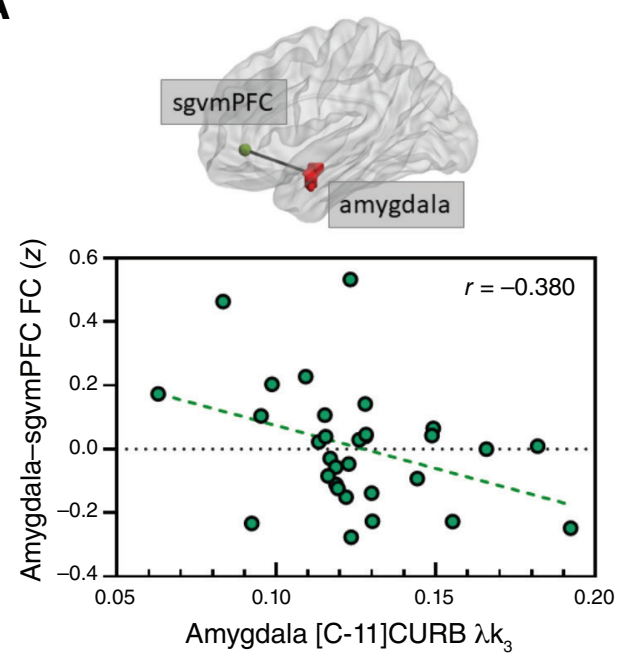

B

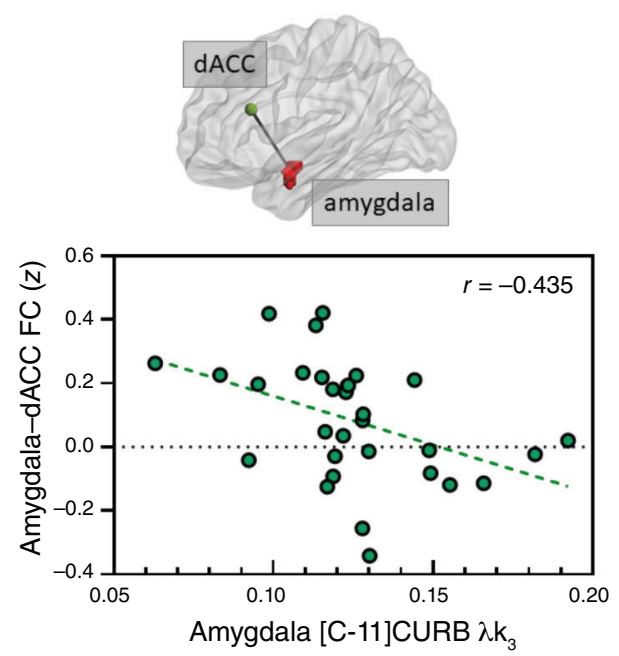

Fig. 1: Scatter plot of correlations in a ROI-to-ROI approach. Amygdala [C-11]CURB $\lambda \mathrm{k}_{3}$ was negatively correlated (Pearson correlation) with the strength of the functional connectivity between the amygdala and $(A)$ the subgenual ventromedial prefrontal cortex and $(B)$ the dorsal anterior cingulate cortex. [C-11]CURB $=[\mathrm{C}-11$-carbonyl]-6-hydroxy-[1,10-biphenyl]-3-yl cyclohexylcarbamate; dACC = dorsal anterior cingulate cortex; $\mathrm{FC}$ = functional connectivity; $\mathrm{ROI}=$ region of interest; sgvmPFC = subgenual ventromedial prefrontal cortex.

A

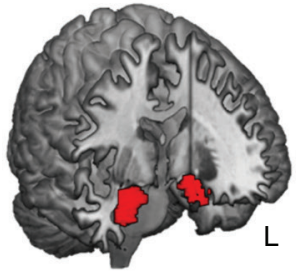

Amygdala ROI
B

vmPFC
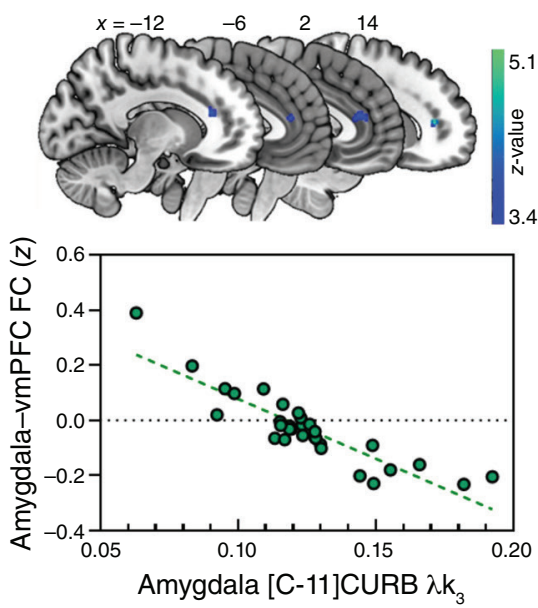

C ITG

Fig. 2: Scatter plot of voxel-wise whole-brain correlations in a seed-to-voxel approach. (A) Bilateral amygdala region of interest. (B) We found a significant negative Pearson correlation between amygdala [C-11]CURB $\lambda \mathrm{k}_{3}$ and the functional connectivity of the amygdala with the ventromedial prefrontal cortex $\left(x, y, z=14,40,10 ; k=280 ; p_{\mathrm{CFWE}}<0.05\right)$. Statistical significance was set at $p_{\mathrm{cFWE}}<0.05$. (C) We found a positive correlation between amygdala [C-11]CURB $\lambda \mathrm{k}_{3}$ and the functional connectivity of the amygdala with the right inferior temporal gyrus $(x, y$, $\left.z=52,-58,-16 ; k=242 ; p_{\text {cFWE }}<0.05\right)$. [C-11]CURB = [C-11-carbonyl]-6-hydroxy-[1,10-biphenyl]-3-yl cyclohexylcarbamate; FC = functional connectivity; cFWE = cluster-level, family-wise error corrected; ITG = inferior temporal gyrus; $\mathrm{ROI}=$ region of interest; vmPFC = ventromedial prefrontal cortex. 
5.12; $p_{\text {cFWE }}<0.05 ;$ Fig. 2B). The cluster identified was adjacent to the pre-selected ROIs but did not overlap with them (Appendix 1, Figure S2). This analysis also revealed a positive correlation between amygdala [C-11]CURB $\lambda \mathrm{k}_{3}$ and amygdala functional connectivity with the right posterior inferior temporal gyrus (ITG; MNI coordinates $x, y, z=52,-58,-16 ; k$ $=242, t=5.99 ; z=4.77 ; p_{\mathrm{cFWE}}<0.05$; Figure $2 \mathrm{C}$ ). Trait neuroticism was not related to amygdala-vmPFC functional connectivity $(r=-0.26 ; p=0.97)$. We also analyzed right and left amygdala ROIs separately, as outlined above, to confirm the functional connectivity results using the bilateral amygdala. The results of a statistical analysis comparing the $\beta$ slopes estimates of the functional connectivity of the left and right amygdala ROIs and amygdala [C-11]CURB $\lambda \mathrm{k}_{3}$ were not significant, suggesting no laterality effect. We further investigated whether [C-11]CURB $\lambda \mathrm{k}_{3}$ in regions outside the amygdala (i.e., the medial prefrontal cortex, cingulate, and striatum) were correlated with amygdalar functional connectivity. Results are reported in Appendix 1, Table S1.

Partial correlations investigating the effects of age, body mass index, sex, ethnicity and smoking did not affect any of the findings listed above for both seed-to-voxel (vmPFC: $r=-0.90, p<0.01$; ITG: $r=0.75, p<0.01)$ and ROI-to-ROI approaches (sgvmPFC: $r=-0.46, p=0.01$; dACC: $r=-0.60$, $p<0.01$ ). After controlling for the FAAH C385A rs324420 genotype $(\mathrm{C} / \mathrm{C}$ v. A/A and $\mathrm{A} / \mathrm{C})$ both the seed-to-voxel (vmPFC: $r=-0.90, p<0.01$; ITG: $r=-0.79, p<0.01$ ) and ROIto-ROI approaches (sgvmPFC: $r=-0.38, p=0.02$; dACC: $r=-0.42, p=0.01)$ remained significant.

We tested whether FAAH C385A genotypes were related to trait neuroticism and amygdala functional connectivity (using functional connectivity z-scores) using a 2-sample $t$ test and found no effect of the C385A genotype on trait neuroticism $(p=0.23)$ or on amygdala functional connectivity with the sgvmPFC ( $p=0.18)$ or dACC ( $p=0.73)$ ROIs, or with the vmPFC $(p=0.91)$ or ITG $(p=0.94)$ clusters.

Differences in [C-11]CURB $\lambda \mathrm{k}_{3}$ across FAAH genotypes are reported in Appendix 1, Table S1.

\section{Discussion}

We provide the first report in humans that levels of the endocannabinoid enzyme FAAH measured in vivo in the brain are negatively correlated with frontoamygdalar functional connectivity. Our findings supported our hypothesis and extend earlier neuroimaging studies in humans. ${ }^{2,15,16}$

This suggests that higher levels of AEA in the brain could increase coupling strength in frontoamygdalar networks, affecting stress response. ${ }^{16}$ Our findings are of relevance to ongoing clinical trials of FAAH inhibitors for disorders of fear regulation (such as PTSD) and suggest that clinical doses of FAAH inhibitors may regulate neural coupling in amygdalar networks in humans, strengthening the empirical evidence for the use of FAAH inhibitors in disorders such as PTSD.

The current in vivo multimodal imaging results were in line with preclinical literature, which reports that genetic and pharmacologic manipulations of FAAH affect the basolateral amygdala (BLA)-medial prefrontal cortex network. ${ }^{5}$ The BLA is rich in FAAH and CB1 receptors; ${ }^{39}$ it receives glutamatergic input from vmPFC pyramidal neurons and relays output to the centromedial nuclei of the amygdala and reciprocal cortical areas. ${ }^{40}$ Exposure to stress has been shown to rapidly increase FAAH-mediated AEA hydrolysis in the BLA, ${ }^{41,42}$ reducing AEA/CB1 signalling and increasing excitation (glutamatergic signalling) of BLA neurons. ${ }^{43}$ Projection neurons from the BLA predominately synapse onto local inhibitory interneurons in the vmPFC, favouring feedforward inhibition onto cortical neurons and reducing neuronal activity in the vmPFC. ${ }^{44}$ This mechanism could reduce activity of cortical projection neurons reciprocally projecting back to the BLA, compromising vmPFC-BLA functional connectivity. Under conditions of sustained stress, protracted loss of AEA signalling in the BLA also favours morphological changes in BLA pyramidal neurons, favouring increased dendritic arborization and spine densities. ${ }^{45}$ As such, inhibition of FAAH and elevations in AEA signalling may help to reduce stress, anxiety and fear by preventing hyperactivity of BLA neurons projecting to the vmPFC. If this is the case, feedforward cortical inhibition would be reduced, promoting frontal cortical control over amygdala activity. More recently, studies of mice with the 385A mutation have also shown that increased fear extinction in mice with the $385 \mathrm{~A}$ variant is coupled with enhanced frontoamygdalar connectivity, a finding that directly aligns with the human data and strongly suggests that reduced fear-relevant behaviour in humans, and in mice with the C385A variant, is likely due to the control of amygdala signalling by prefrontal cortex. ${ }^{14,16}$

Although the action of AEA is not restricted to CB1 receptors (AEA also binds CB2 receptors, GPR55 and transient receptor potential vanilloid type 1 ), the findings described above are believed to be dependent on CB1 receptor activation, because the effects of FAAH inhibitors on stress-induced changes are eliminated by CB1 antagonists. ${ }^{46}$ Despite this, the contribution of other FAAH substrates (oleoylethanolamine, palmitoylethanolamide, dehydroepiandrosterone) and other receptors as potential mechanisms for the effects reported are largely uncharacterized and cannot be ruled out. Furthermore, although our study focused on FAAH levels in the amygdala, it is unclear whether the impact of FAAH on functional connectivity is selective to FAAH levels in amygdala in other regions or in whole brain (Appendix 1, Table S1).

Although the dACC, sgvmPFC and amygdala are key areas recruited during emotion processing and are coactivated during socioaffective tasks, they are also conceptualized as being part of the default mode network. ${ }^{47}$ In this regard, large meta-analytic studies have shown positive coactivation at rest of the amygdala with the dACC and in some, with the vmPFC. ${ }^{48,49}$ In the current study we found that hemodynamics in the bilateral amygdala positively mapped onto a large cluster of voxels in the anterior cingulate cortex (ACC) and bilateral precentral gyrus. Although the identified cluster using a whole-brain approach was adjacent to the preselected dACC ROI, we did not find robust significant functional connectivity with this region or with the sgvmPFC. However, using a more liberal threshold (at an uncorrected 
voxel-level $p<0.01$ and cluster-size uncorrected $p<0.05$ ) the functional connectivity map extended to include preselected ROIs as well as the seed-to-voxel identified cluster (data not shown), suggesting that we may have been underpowered to detect a significant effect. Despite the absence of significant amygdala functional connectivity, the ROI and wholebrain functional connectivity data correlated significantly with FAAH activity.

The anatomic extent of our findings aligned with the results of retrograde tracing studies in primates, which suggest reciprocal connections between the basolateral amygdala and pyramidal neurons (layer V) in the ACC (BA 24), infralimbic cortex (BA 25) and prelimbic area (BA 32). ${ }^{50,51}$ Our ROI-to-ROI findings replicated both the findings of Dincheva and colleagues ${ }^{16}$ in the sgvmPFC and those of Gärtner and colleagues in the dACC. ${ }^{15}$ However, the more conservative seed-to-voxel analysis identified a cluster in the vmPFC that included the $\mathrm{dACC}$ (BA 32) and the rostral portion of the frontal lobe and ACC (BA 24), but not the sgvmPFC (BA 25). The discrepancy between the ROI-to-ROI and seed-to-voxel approaches may have been due to differences in the statistical threshold (the seed-to-voxel approach being more conservative than the ROI-to-ROI approach). Although our results largely suggest that amygdala-vmPFC functional connectivity and brain FAAH levels are linked, the exact regional extent of the findings remains uncertain. The vmPFC includes subregions (infralimbic, prelimbic and ACC) with distinct cytoarchitecture and connectivity patterns, and which have been differentially implicated in emotion regulation and fear-related behaviours. ${ }^{17,52}$ It is unclear based on our results in humans whether a differing role for FAAH exists across subregions of the vmPFC, particularly the ACC and sgvmPFC.

The amygdala-ITG (occipitotemporal BA 37) functional connectivity related to FAAH in brain was a new finding. Anatomically, the amygdala comprises numerous nuclei..$^{53}$ Projections from the occipitotemporal lobe and lateral nucleus of the amygdala have been identified using autoradiographic techniques..$^{50}$ The lack of precision in selecting the whole amygdala as our seed region could partially explain our results in the ITG. However, examination of the overlap between our amygdala mask and the amygdala probabilistic cytoarchitectonic atlas suggests that most of the signal arising from the automated anatomic labelling ROIs is from the basolateral nucleus. ${ }^{54}$ This finding needs to be explored further, particularly in light of a recent report suggesting impaired amygdala-ITG functional connectivity in people with depression. ${ }^{55}$

With the current small sample size, we did not find that differences in functional connectivity were related to $F A A H$ genotype; however, the finding that both the ROI-to-ROI and seed-to-voxel analyses survived after controlling for genetic variability in FAAH levels suggests that direct measurement of FAAH levels, which may be influenced by epigenetic changes and the environment, brings precision to the development of brain-based endophenotypes.

Previous literature has suggested that neuroticism is associated with altered functional connectivity in amygdalarelated circuits, ${ }^{56-58}$ and that low levels of endocannabinoids in this circuit increase proneness to anxiety and negative emotions. ${ }^{42}$ Our failure to find a relationship with selfreported trait neuroticism may be an issue of power, particularly when considering that sex, age and factors affecting state anxiety may also modulate the effect. Future studies in larger samples are needed to examine whether the relationship between FAAH levels and frontoamygdalar functional connectivity affect state and trait anxiety as well as fear and emotion processing.

\section{Limitations}

Our study had several limitations, including a small sample size, limited behavioural assessments and the inclusion of tobacco smokers, which may have affected frontoamygdalar coupling ${ }^{59}$ or brain AEA. ${ }^{38}$

\section{Conclusion}

Our findings that lower FAAH levels in brain measured through molecular imaging correlated with the strength of functional connectivity in frontoamygdalar circuits in humans support the view that FAAH inhibition could alter functional connectivity in frontoamygdalar circuits in humans, regulating stress and affect.

Affiliations: From the Addiction Imaging Research Group, Toronto, Ont., Canada (Green, Westwood, Boileau); the Human Brain Lab, Toronto, Ont., Canada (Kish, Tong, McCluskey); the Campbell Family Mental Health Research Institute, Ont., Canada (Kim, Tyndale, Strafella, Houle, Lobaugh, Boileau); the Brain Health Imaging Centre, Centre for Addiction and Mental Health, Toronto, Ont., Canada (Kim, Kish, Strafella, Tong, McCluskey, Houle, Lobaugh); the Departments of Psychiatry, Institute of Medical Sciences, University of Toronto, Toronto, Ont., Canada (Kish, Tyndale, Strafella, Houle, Boileau); the Department of Pharmacology \& Toxicology, University of Toronto, Toronto, Ont., Canada (Kish, Tyndale); the Institute of Medical Sciences, University of Toronto, Toronto, Ont., Canada (Green, Kish, Houle, Lobaugh, Boileau); the Hotchkiss Brain Institute and Mathison Centre for Mental Health Research and Education, Departments of Cell Biology and Anatomy \& Psychiatry, University of Calgary, Calgary, Alta., Canada (Hill); the Morton and Gloria Shulman Movement Disorder Unit and E.J. Safra Parkinson Disease Program, Toronto Western Hospital, UHN, University of Toronto, Toronto, Ont., Canada (Strafella); and the Division of Neurology, Faculty of Medicine, University of Toronto, Toronto, Ont., Canada (Lobaugh).

Funding: This study was supported in part by Canada Foundation for Innovation, the Ontario Ministry of Research and Innovation (S. Houle), The National Institute of Health and National Institute of Drug Abuse (NIH/NIDA R21 DA036024; I. Boileau), Canadian Institutes of Health Research FDN-154294 (R. Tyndale), and a Canada Research Chair in Pharmacogenomics (R. Tyndale).

Competing interests: M. Hill has served on scientific advisory boards for Lundbeck and Sophren Therapeutics. R. Tyndale has consulted for Quinn Emanuel and Ethismos on unrelated topics. No other competing interests declared.

Contributors: R. Tyndale, D. Westwood and I. Boileau designed the study. J. Kim, R. Tyndale, T. McCluskey, D. Westwood and I. Boileau acquired the data, which D. Green, J. Kim, S. Kish, R. Tyndale, M. Hill, A. Strafella, J. Tong, T. McCluskey, N. Lobaugh and I. Boileau analyzed. D. Green, J. Kim, D. Westwood, N. Lobaugh and I. Boileau wrote the article, which all authors reviewed. All authors approved the final version to be published and 
can certify that no other individuals not listed as authors have made substantial contributions to the paper.

Content licence: This is an Open Access article distributed in accordance with the terms of the Creative Commons Attribution (CC BY-NC-ND 4.0) licence, which permits use, distribution and reproduction in any medium, provided that the original publication is properly cited, the use is noncommercial (i.e., research or educational use), and no modifications or adaptations are made. See: https:/ / creativecommons.org/licenses/by-nc-nd/4.0/

\section{References}

1. Stein MB, Simmons AN, Feinstein JS, et al. Increased amygdala and insula activation during emotion processing in anxiety-prone subjects. Am J Psychiatry 2007;164:318-27.

2. Hariri AR, Gorka A, Hyde LW, et al. Divergent effects of genetic variation in endocannabinoid signaling on human threat- and reward-related brain function. Biol Psychiatry 2009;66:9-16.

3. Swartz JR, Knodt AR, Radtke SR, et al. A neural biomarker of psychological vulnerability to future life stress. Neuron 2015;85:505-11.

4. Gunduz-Cinar O, Hill MN, McEwen BS, et al. Amygdala FAAH and anandamide: mediating protection and recovery from stress. Trends Pharmacol Sci 2013;34:637-44.

5. Gunduz-Cinar O, Hill MN, McEwen BS, et al. Amygdala FAAH and anandamide: mediating protection and recovery from stress. Trends Pharmacol Sci 2013;34:637-44.

6. Mayo LM, Asratian A, Lindé J, et al. Elevated anandamide, enhanced recall of fear extinction, and attenuated stress responses following inhibition of fatty acid amide hydrolase: a randomized, controlled experimental medicine trial. Biol Psychiatry 2020; 87:538-547.

7. Chiang KP, Gerber AL, Sipe JC, et al. Reduced cellular expression and activity of the P129T mutant of human fatty acid amide hydrolase: evidence for a link between defects in the endocannabinoid system and problem drug use. Hum Mol Genet 2004;13:2113-9.

8. Sipe JC, Chiang K, Gerber AL, et al. A missense mutation in human fatty acid amide hydrolase associated with problem drug use. Proc Natl Acad Sci U S A 2002;99:8394-9.

9. Boileau I, Tyndale RF, Williams B, et al. The fatty acid amide hydrolase C385A variant affects brain binding of the positron emission tomography tracer [11C]CURB. J Cereb Blood Flow Metab 2015;35:1237-40.

10. Cajanus K, Holmström EJ, Wessman M, et al. Effect of endocannabinoid degradation on pain: role of FAAH polymorphisms in experimental and postoperative pain in women treated for breast cancer. Pain 2016;157:361-9.

11. Habib AM, Okorokov AL, Hill MN, et al. Microdeletion in a FAAH pseudogene identified in a patient with high anandamide concentrations and pain insensitivity. Br J Anaesth 2019;123:e249-53.

12. Hariri AR, Gorka A, Hyde LW, et al. Divergent effects of genetic variation in endocannabinoid signaling on human threat- and reward-related brain function. Biol Psychiatry 2009;66:9-16.

13. Gunduz-Cinar O, MacPherson KP, Cinar R, et al. Convergent translational evidence of a role for anandamide in amygdalamediated fear extinction, threat processing and stress-reactivity. Mol Psychiatry 2013;18:813-23.

14. Gee DG, Fetcho RN, Jing D, et al. Individual differences in frontolimbic circuitry and anxiety emerge with adolescent changes in endocannabinoid signaling across species. Proc Natl Acad Sci U S A 2016;113:4500-5

15. Gärtner A, Dörfel D, Diers K, et al. Impact of FAAH genetic variation on fronto-amygdala function during emotional processing. Eur Arch Psychiatry Clin Neurosci 2019;269:209-21.

16. Dincheva I, Drysdale AT, Hartley CA, et al. FAAH genetic variation enhances fronto-amygdala function in mouse and human. Nat Commun 2015;6:6395.

17. Rea K, McGowan F, Corcoran L, et al. The prefrontal cortical endocannabinoid system modulates fear-pain interactions in a subregionspecific manner. Br J Pharmacol 2019;176:1492-505.

18. Etkin A, Egner T, Kalisch R. Emotional processing in anterior cingulate and medial prefrontal cortex. Trends Cogn Sci 2011;15:85-93.

19. Wilson AA, Garcia A, Parkes J, et al. [11C]CURB: evaluation of a novel radiotracer for imaging fatty acid amide hydrolase by positron emission tomography. Nucl Med Biol 2011;38:247-53.
20. Rusjan PM, Wilson AA, Mizrahi R, et al. Mapping human brain fatty acid amide hydrolase activity with PET. J Cereb Blood Flow Metab 2013;33:407-14.

21. Boileau I, Rusjan PM, Williams B, et al. Blocking of fatty acid amide hydrolase activity with PF-04457845 in human brain: a positron emission tomography study with the novel radioligand [11C] CURB. J Cereb Blood Flow Metab 2015;35:1827-35.

22. Boileau I, Mansouri E, Williams B, et al. Fatty acid amide hydrolase binding in brain of cannabis users: imaging with the novel radiotracer [11C]CURB. Biol Psychiatry 2016;80:691-701.

23. First MB, Gibbon M, Spitzer RL et al. Structured clinical interview for DSM-IV axis II personality disorders (SCID-II). Washington (DC): American Psychiatric Press; 1997.

24. Wilson AA, Garcia A, Parkes J, et al. [11C]CURB: evaluation of a novel radiotracer for imaging fatty acid amide hydrolase by positron emission tomography. Nucl Med Biol 2011;38:247-53.

25. Rusjan P, Mamo D, Ginovart N, et al. An automated method for the extraction of regional data from PET images. Psychiatry Res Neuroimaging 2006;147:79-89.

26. Glover GH, Law CS. Spiral-in/out BOLD fMRI for increased SNR and reduced susceptibility artifacts. Magn Reson Med 2001; 46:515-22.

27. Salimi-Khorshidi G, Douaud G, Beckmann CF, et al. Automatic denoising of functional MRI data: combining independent component analysis and hierarchical fusion of classifiers. Neuroimage 2014;90:449-68.

28. Smith SM. Fast robust automated brain extraction. Hum Brain Mapp 2002;17:143-55.

29. Jenkinson M, Bannister P, Brady M, et al. Improved optimization for the robust and accurate linear registration and motion correction of brain images. Neuroimage 2002;17:825-41.

30. Calhoun VD, Wager TD, Krishnan A, et al. The impact of $T_{1}$ versus EPI spatial normalization templates for fMRI data analyses. Hum Brain Mapp 2017;38:5331-42.

31. Behzadi Y, Restom K, Liau J, et al. A component based noise correction method (CompCor) for BOLD and perfusion based fMRI. Neuroimage 2007;37:90-101.

32. Van Dijk KRA, Sabuncu MR, Buckner RL. The influence of head motion on intrinsic functional connectivity MRI. Neuroimage 2012;59:431-8

33. Maldjian JA, Laurienti PJ, Kraft RA, et al. An automated method for neuroanatomic and cytoarchitectonic atlas-based interrogation of fMRI data sets. Neuroimage 2003;19:1233-9.

34. Worsley KJ, Marrett S, Neelin P, et al. A unified statistical approach for determining significant signals in images of cerebral activation. Hum Brain Mapp 1996;4:58-73.

35. Maccarrone M, Valverde O, Barbaccia ML, et al. Age-related changes of anandamide metabolism in CB1 cannabinoid receptor knockout mice: correlation with behaviour. Eur J Neurosci 2002; 15:1178-86.

36. Riebe CJN, Hill MN, Lee TTY, et al. Estrogenic regulation of limbic cannabinoid receptor binding. Psychoneuroendocrinology 2010;35:1265-9.

37. Touriño C, Oveisi F, Lockney J, et al. FAAH deficiency promotes energy storage and enhances the motivation for food. Int J Obes (Lond) 2010;34:557-68.

38. Cippitelli A, Astarita G, Duranti A, et al. Endocannabinoid regulation of acute and protracted nicotine withdrawal: effect of FAAH inhibition. PLoS One 2011;6:e28142.

39. Ramikie TS, Patel S. Endocannabinoid signaling in the amygdala: anatomy, synaptic signaling, behavior, and adaptations to stress. Neuroscience 2012;204:38-52.

40. Price JL, Russchen FTAD. The limbic region. II. The amygdaloid complex. In: Hokfelt BT, Swanson LW, editors. Handbook of chemical neuroanatomy. Amsterdam: Elsevier; 1987; 279-388.

41. Gray JM, Vecchiarelli HA, Morena M, et al. Corticotropinreleasing hormone drives anandamide hydrolysis in the amygdala to promote anxiety. J Neurosci 2015;35:3879-92.

42. Hill MN, McLaughlin RJ, MoMrrish AC, et al. Suppression of amygdalar endocannabinoid signaling by stress contributes to activation of the hypothalamic-pituitary-adrenal axis. Neuropsychopharmacology 2009;34:2733-45.

43. Yasmin F, Colangeli R, Morena M, et al. Stress-induced modulation of endocannabinoid signaling leads to delayed strengthening of synaptic connectivity in the amygdala. Proc Natl Acad Sci U S A 2020;117:650-5. 
44. Gabbott PLA, Warner TA, Busby SJ. Amygdala input monosynaptically innervates parvalbumin immunoreactive local circuit neurons in rat medial prefrontal cortex. Neuroscience 2006;139:1039-48

45. Hill MN, Kumar SA, Filipski SB, et al. Disruption of fatty acid amide hydrolase activity prevents the effects of chronic stress on anxiety and amygdalar microstructure. Mol Psychiatry 2013;18:1125-35.

46. Danandeh A, Vozella V, Lim J, et al. Effects of fatty acid amide hydrolase inhibitor URB597 in a rat model of trauma-induced long-term anxiety. Psychopharmacology (Berl) 2018;235:3211-21.

47. Schilbach L, Eickhoff SB, Rotarska-Jagiela A, et al. Minds at rest? Social cognition as the default mode of cognizing and its putative relationship to the "default system" of the brain. Conscious Cogn 2008; 17:457-67.

48. Amft M, Bzdok D, Laird AR, et al. Definition and characterization of an extended social-affective default network. Brain Struct Funct 2015;220:1031-49.

49. Marusak HA, Thomason ME, Peters C, et al. You say "prefrontal cortex" and I say "anterior cingulate": meta-analysis of spatial overlap in amygdala-to-prefrontal connectivity and internalizing symptomology. Transl Psychiatry 2016;6:e944.

50. Pandya DN, Van Hoesen GW, Mesulam MM. Efferent connections of the cingulate gyrus in the rhesus monkey. Exp Brain Res 1981; 42:319-30.

51. Carmichael ST, Price JL. Limbic connections of the orbital and medial prefrontal cortex in macaque monkeys. I Comp Neurol 1995;363:615-41
52. Vidal-Gonzalez I, Vidal-Gonzalez B, Rauch SL, et al Microstimulation reveals opposing influences of prelimbic and infralimbic cortex on the expression of conditioned fear. Learn Mem 2006;13:728-33.

53. McDonald AJ, Mascagni F. Projections of the lateral entorhinal cortex to the amygdala: a Phaseolus vulgaris leucoagglutinin study in the rat. Neuroscience 1997;77:445-59.

54. Amunts K, Kedo O, Kindler M, et al. Cytoarchitectonic mapping of the human amygdala, hippocampal region and entorhinal cortex: intersubject variability and probability maps. Anat Embryol (Berl) 2005:210:343-52.

55. Cheng W, Rolls ET, Qiu J, et al. Functional connectivity of the human amygdala in health and in depression. Soc Cogn Affect Neurosci 2018;13:557-68.

56. Silverman MH, Wilson S, Ramsay IS, et al. Trait neuroticism and emotion neurocircuitry: functional magnetic resonance imaging evidence for a failure in emotion regulation. Dev Psychopathol 2019;31:1085-99.

57. Deng Y, Li S, Zhou R, et al. Neuroticism modulates the functional connectivity from amygdala to frontal networks in females when avoiding emotional negative pictures. Front Behav Neurosci 2019;13:102.

58. Wang Y, Zhu Y, Chen P, et al. Neuroticism is associated with altered resting-state functional connectivity of amygdala following acute stress exposure. Behav Brain Res 2018;347:272-80.

59. Shen $Z$, Huang $P$, Wang $C$, et al. Altered function but not structure of the amygdala in nicotine-dependent individuals. Neuropsychologia 2017;107:102-7. 